\title{
Detecting Fake Face Input for Face Authentication by DCT with Compensating the Main Spindle Position of Face
}

\author{
Ryo Furuiea,, ${ }^{\mathrm{a}}$, Yuji Goda ${ }^{\mathrm{a}}$, Lifeng Zhang ${ }^{\mathrm{a}}$ \\ ${ }^{a} 1-1$ sensui-cho Tobata-ku, Kitakyushu city, Fukuoka, Japan 804-8550 \\ Kyusyu Institute of Technology \\ *Corresponding Author: p349430r@mail.kyutech.
}

\begin{abstract}
In this information age, biometrics has been used in many fields in our social life. Face as a very important individual feature has been researched for a long time for individual authentication. It takes the advantage that can be captured from a long distance and can be performed without notifying the subject. But face is exposed almost publicly, and can be caught easily by other people, the problem that people take authentication illegally with other's face photo is appeared. In order to prevent such a happening, detecting fake face input for face authentication by DCT with compensating the main spindle position of face is proposed here. In this paper the dynamic difference between real face and face photo has been studied. Simulation result shows that the proposed approach can distinguish the fake face input to prevent masquerading, either the face picture is fixed or moving which the previous research cannot do.
\end{abstract}

Keywords: Authentication, Masquerading, DCT, main spindle position of face

\section{Introduction}

Today, the information-oriented society advances more and more. The popularization of network services is realized in each field of social life. Personal authentication is used everywhere. For example, entering a building, accessing an important department of company, withdraw the deposit by cash card from a bank ATM, and the demand increases day by day. There are possession authentication, intellectual authentication and biometrics authentication in personal authentications.

\section{Possession authentication}

Personalized possession is used to this authentication method. The possession is a kind of information media, ordinary shaped as chip cards or magnetic cards with authentication information on it. We can suppose it is lost or stolen when the possessions are disappeared. But we must always carry it, and we can lend it to others.

\section{Intellectual authentication}

Password is most used to this kind of authentication. Normally, the password is secret information to other people. But the biggest problem is we cannot aware if other people know it until the damage happened. The best way to keep the password is just remember it in mind, but in fact people always write down it on a note or somewhere for the case of forgotten. And this causes an extra risk for leak.

\section{Biometrics authentication}

In the past decade, biometric authentication has been widely researched and applied. This approach use human body features as authentication subject. Fingerprint, iris, vein pattern and face are most popular biometrics. Using this method, people do not need to remember any thing so there are no forgotten happenings. But the disadvantage is the 
trade off between accuracy and performance is difficult to be decided. Such biometrics is unique to an individual, but the shapes are slightly changed according to the body condition or age. This means a deformed biometric image might pass the security check.

As the information for possession authentication and intellectual authentication might lost or stolen, biometrics take the advantage of individual specified information. So we think that the future society will regard the biometrics authentication as important approach of security. Now, the security industry focuses on face authentication. Because the method does not require the cooperation of the object and it can identify user from a distance. It's a system that automatically determines the person from a digital image. This method is easy to identify the original person when there isn't any illegal input usage. But the method has a defect in terms of a security that the method authenticate users picture. In order to solve this problem, we focus on a difference between the movement of face picture and real face. We propose a new face determination method by taking a difference between current frame and before frame.

Now, face authentication use recognition system with Karhunen-Loeve transformation (KLT) and discrete cosine transformation (DCT). KLT and DCT are amount of characteristic in order to recognize face. KLT is optimum orthogonal expansion in a standard of minimum mean square error. Character recognition uses those, and their recognition rate is high. But the calculation is difficult. So we expect to accelerate the transformation by decode into hardware. But technical experts regard it as taking a long time. On the other hand, DCT is similar to KLT's transfer characteristic, and it has been found that it can calculate with a simple process. So technical experts use this method as an optimal image coding system. Authentication method with DCT use encoded information, but its quality is higher than other orthogonal transform.

As a result, we think that DCT is correct in face authentications. Our research can distinguish the face picture form real face with DCT. Previous research is similar to this research when the illegal input face picture is fixed, but it

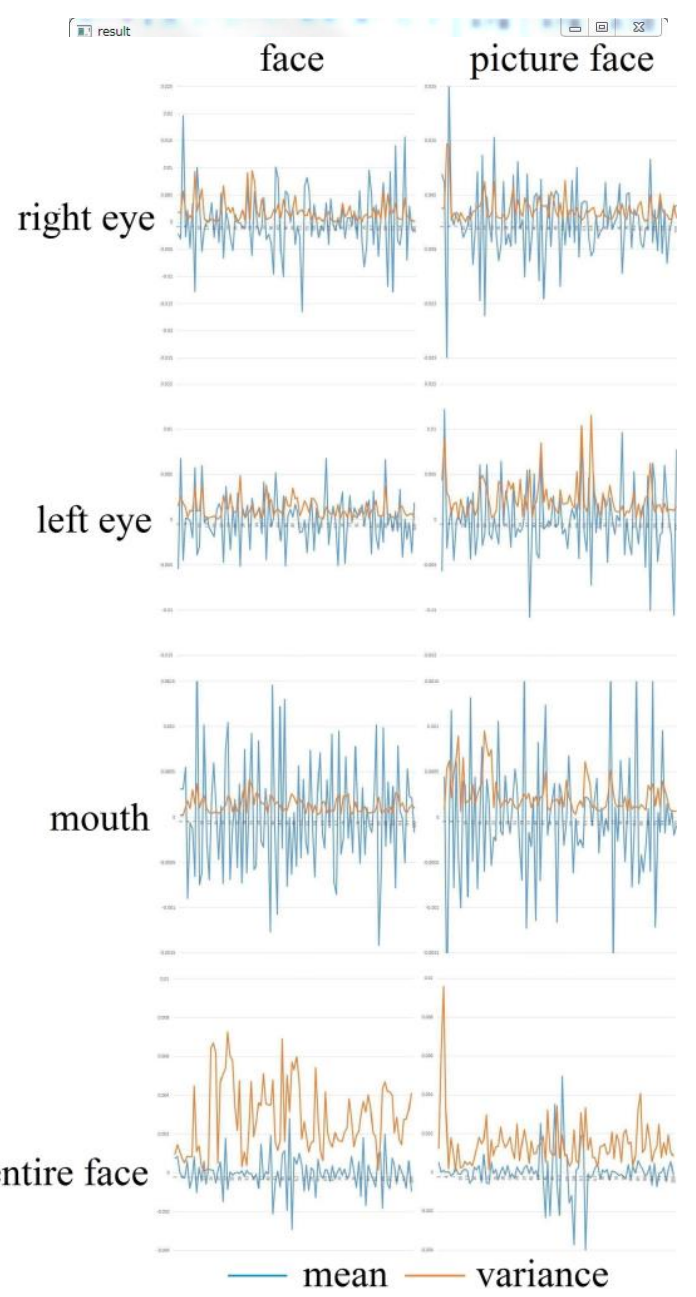

Fig. 1 before

doesn't work well if the face picture moving. So we aim at improvement of accuracy and performance.

\section{Theory}

The basic thinking is if the input is a face picture, the feature point like eyes and mouth are invariable, this means if the image keeps still, the difference of neighbor frames is zero. But the real face input changing timely, the difference of neighbor frames is not zero. Therefore, we can distinguish the face picture from the real face. Below is the explanation of this characteristic between tow kinds of inputs.

\subsection{Discriminate between face picture and real face}

This research makes use of a facial motion for several seconds. The system detects face position first and then extracts the feature area (mouth, eyes) for each frame, and takes the difference between current frame and before frame 
in each part. By calculating the mean and variance of the difference of DCT transform, the change of two frames is intelligibly shown.

The mean is fundamental statistics, which show a center location of a date. In this research the mean $\bar{x}$ is calculated by Eq.(1).

$$
\bar{x}=\frac{1}{N} \sum_{i=1}^{N} x_{i} \quad(i=1,2, \ldots N)
$$

Where $x_{i}$ is the difference value of DCT components, $\mathrm{N}$ is total number of DCT components, and at here it equals to the pixel number.

The variance value shows the scatter of a date and is calculate as below:

$$
V=\frac{1}{N} \sum_{i=1}^{N}\left(\bar{x}-x_{i}\right)^{2}(i=1,2, \ldots N)
$$

Our research takes the difference between current frame and previous frame. Thus, we can recognize the variance of difference between current frame and previous frame in the entire image. Furthermore, we can use the difference to discriminate the real face and face picture.

\section{Method}

\section{Compensation of the main spindle position of face}

First of all, we detect eyes. And we detect the slope of two eyes by the eye coordinates. This slope is perpendicular to the main axis of face. So we modify the slope to horizontal direction, therefore the face of the spindle is kept constant straight up by using this method. Fig. 2 shows the picture of before processing, and Fig.3 shows the picture of after

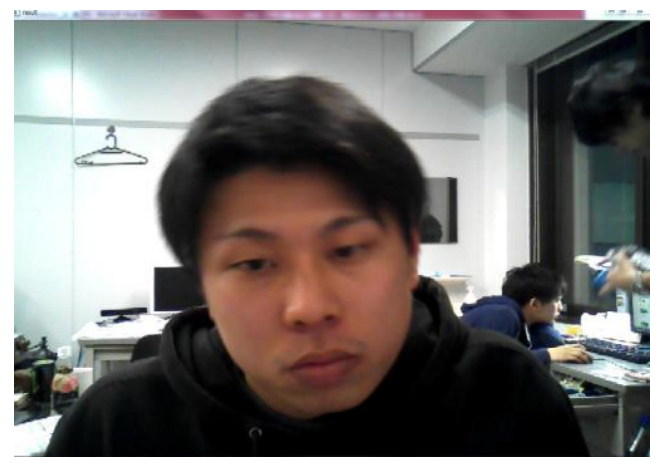

Fig. 2 before processing

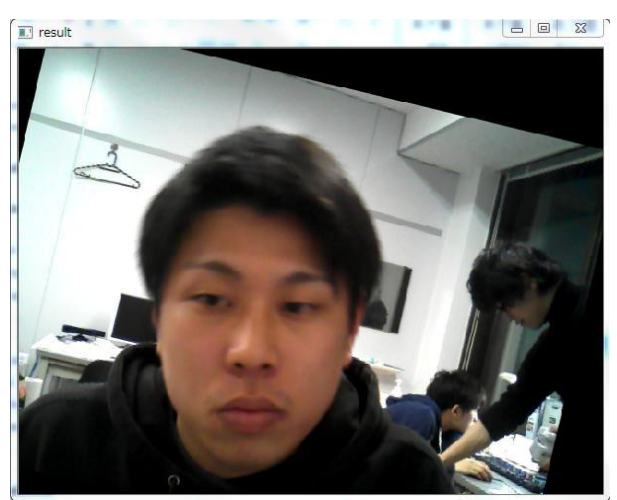

Fig.3 after processing

processing.

\section{Simulation}

We developed a system which improve the discrimination performance between face picture and real face. And verify the reliability of our method by comparing the traditional research and proposed one.

\section{Experimentation result of previous method}

In order to verify the accuracy and performance of previous method ${ }^{(4)}$, we move the face picture when we perform the authentication. Table. 1 and 2 show the compared result of picture face and real face. From these results, the previous method can't discriminate picture face and real face.

Table 1. Sum of the mean

\begin{tabular}{|c|c|c|c|c|}
\hline & Right eye & Left eye & Mouth & Face \\
\hline Person & 0.008071 & 0.002403 & 0.000233 & 0.000752 \\
\hline Picture & 0.008757 & -0.016441 & -0.000367 & 0.004234 \\
\hline
\end{tabular}

Table 2. Sum of the variance

\begin{tabular}{|c|c|c|c|c|}
\hline & Right eye & Left eye & Mouth & Face \\
\hline Person & 0.332591 & 0.191598 & 0.013247 & 0.24655 \\
\hline picture & 0.313349 & 0.310334 & 0.025703 & 0.245858 \\
\hline
\end{tabular}

Because we intended that the method's accuracy improvement by correcting inclination of face. We used webcam (BSW13K05H of BUFFALO). Its pixel number is 1.3 million, and its maximum resolution is $1280 * 1024$ and its maximum frame late is maximum 30fps.

This verification uses input movies of three people (A, $\mathrm{B}, \mathrm{C})$, and uses only low frequency circuit region after carries 
out DCT on the frame, because of consider the property and result of previous study. And the verification compare face picture and face in previous research and proposed method.

\section{Result}

We show the result of each part. We ignored the first several frames, because a computer and camera aren't steady in first several frames. We show the result with mean and variance in order to statistically compare them. As described above, the result approaches 0 , and it shows that the motion of inter-frame is a little. Therefore if face picture is to be in the frame, the result can approach 0 as it is good.

\section{Incremental difference of right eye}

We verify our method in eyes of three people. Table 3, 4 and Fig.4 show the result of incremental difference, the sum of the mean in each frame and the sum of the variance in each frame.

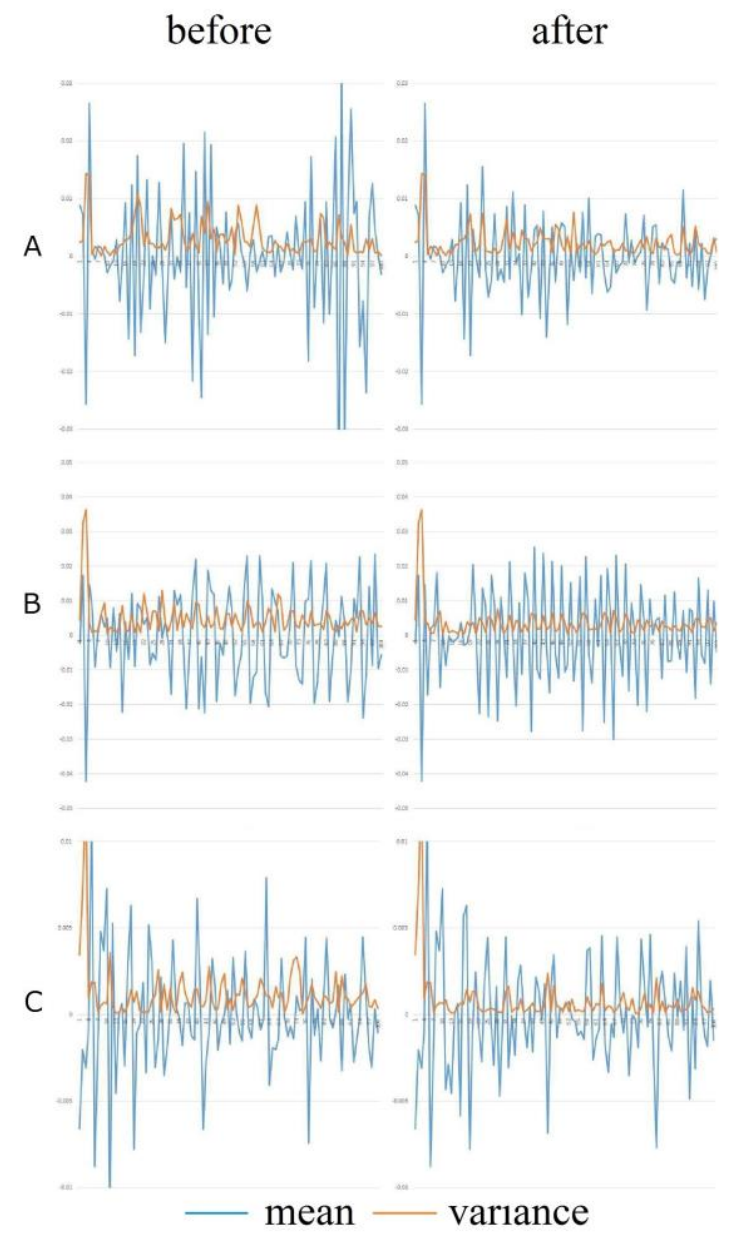

Table 3. Sum of the mean

\begin{tabular}{|c|c|c|c|}
\hline & A & B & C \\
\hline Before & 0.008757 & 0.001816 & -0.00966 \\
\hline After & 0.007081 & -0.023253 & -0.005687 \\
\hline
\end{tabular}

Table 4. Sum of the variance

\begin{tabular}{|c|c|c|c|}
\hline & A & B & C \\
\hline Before & 0.313349 & 0.499261 & 0.125511 \\
\hline After & 0.234716 & 0.37003 & 0.081588 \\
\hline
\end{tabular}

\section{Incremental difference of left eye}

We verify our method in eyes of three people. Table 5, 6 and Fig.5 show the result of incremental difference, the sum of the mean in each frame and the sum of the variance in each frame.

before after

A
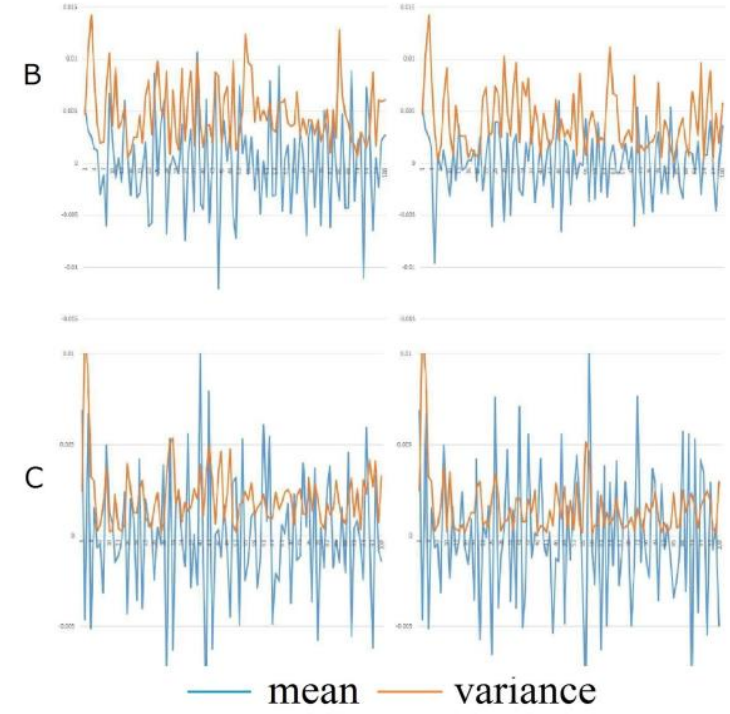

Fig.5 left eye

Fig.4 right eye 
Table 5. Sum of the mean

\begin{tabular}{|c|c|c|c|}
\hline & A & B & C \\
\hline Before & -0.016441 & 0.010907 & 0.000849 \\
\hline After & 0.002083 & 0.003677 & -0.001293 \\
\hline
\end{tabular}

Table 6. Sum of the variance

\begin{tabular}{|c|c|c|c|}
\hline & A & B & C \\
\hline Before & 0.310334 & 0.489577 & 0.215094 \\
\hline After & 0.229761 & 0.404121 & 0.151958 \\
\hline
\end{tabular}

\section{Incremental difference of mouth}

We verify our method in mouths of three people. Table 7, 8 and Fig6 show the result of incremental difference, the sum of the mean in each frame and the sum of the variance in each frame.

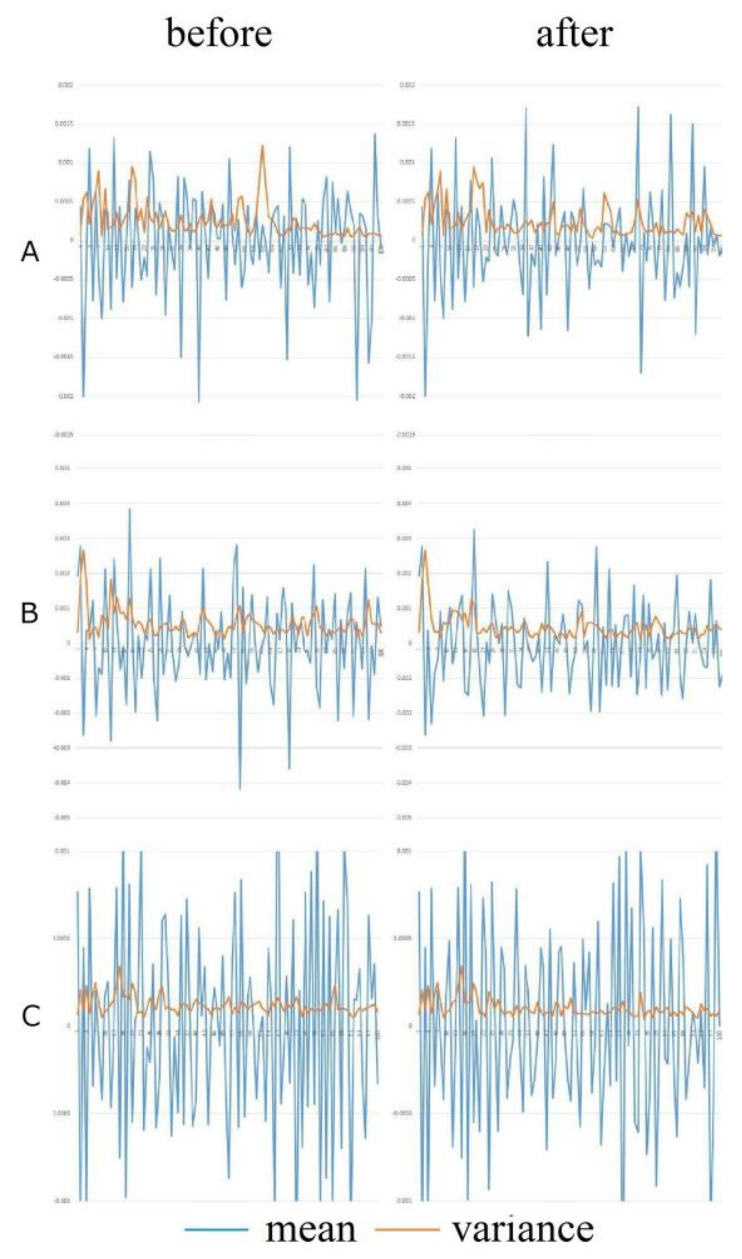

Fig.6 mouth
Table 7. Sum of the mean

\begin{tabular}{|c|c|c|c|}
\hline & A & B & C \\
\hline Before & -0.000367 & 0.001736 & 0.000354 \\
\hline After & -0.000734 & -0.000848 & $0.00 \mathrm{E}+00$ \\
\hline
\end{tabular}

Table 8. Sum of the variance

\begin{tabular}{|c|c|c|c|}
\hline & A & B & C \\
\hline Before & 0.025703 & 0.058476 & 0.012151 \\
\hline After & 0.024294 & 0.047399 & 0.010284 \\
\hline
\end{tabular}

\section{Incremental difference of face}

We verify our method in faces of three people. Table 9, 10 and Fig7 show the result of incremental difference, the sum of the mean in each frame and the sum of the variance in each frame.

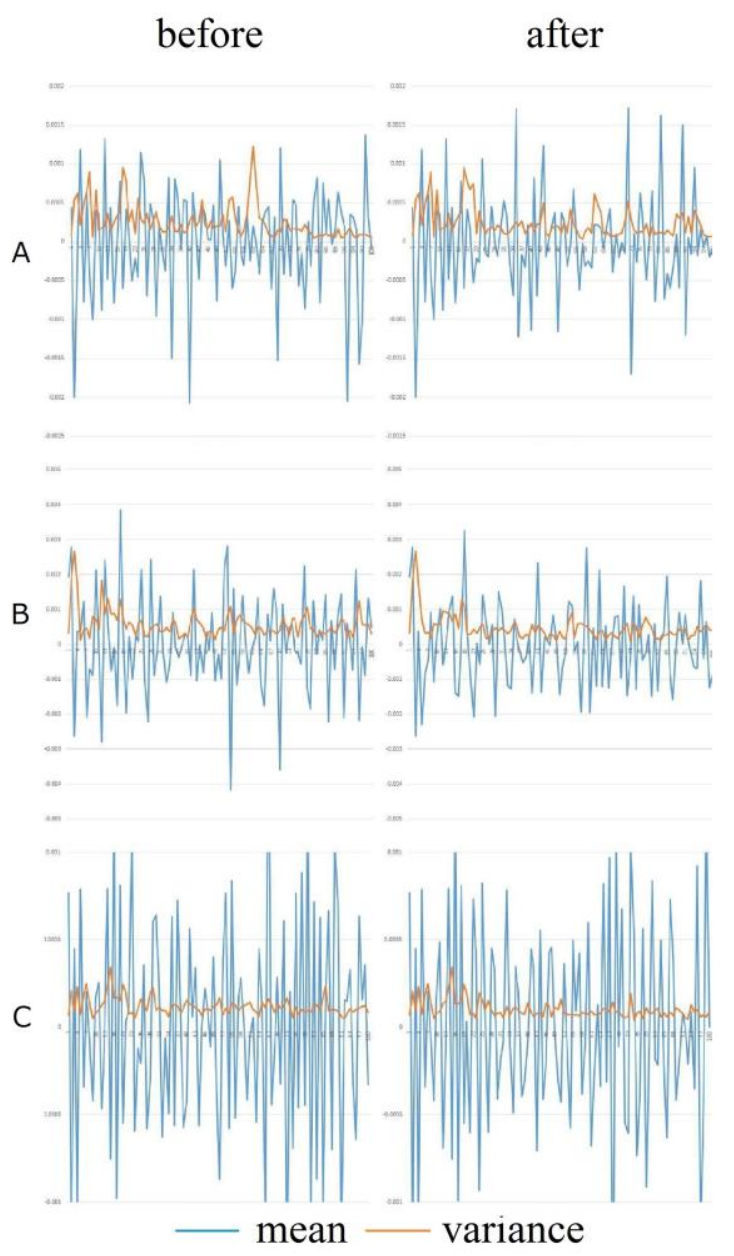

Fig.7 mouth 
Table 9. Sum of the mean

\begin{tabular}{|c|c|c|c|}
\hline & A & B & C \\
\hline Before & 0.004234 & -0.000216 & -0.002726 \\
\hline After & 0.001185 & 0.00022 & 0.001079 \\
\hline
\end{tabular}

Table 10. Sum of the variance

\begin{tabular}{|c|c|c|c|}
\hline & A & B & C \\
\hline Before & 0.245858 & 0.297949 & 0.270322 \\
\hline After & 0.148688 & 0.198313 & 0.139602 \\
\hline
\end{tabular}

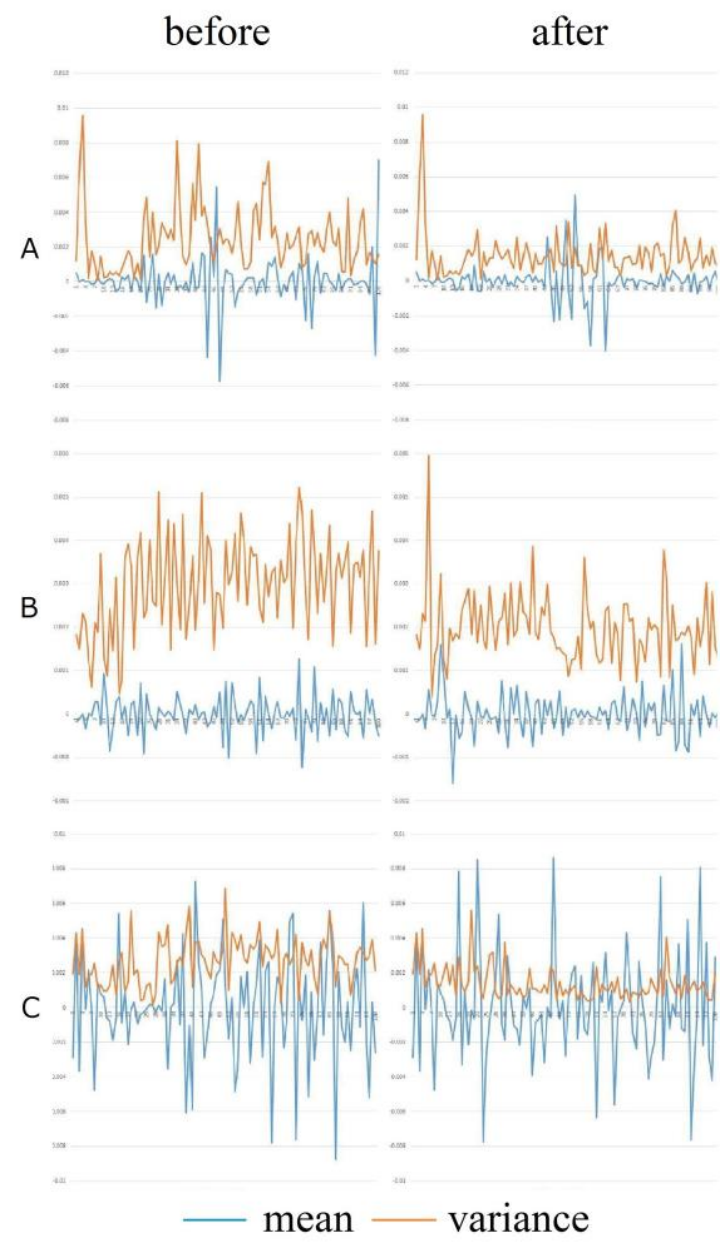

Fig.8 entire face

\section{Comparison between real face and face picture}

We verify the comparison result between a real person face and his picture. Table 11, 12 and Fig. 8 show the result of the comparison in our method.
Table11. Sum of the mean

\begin{tabular}{|c|c|c|c|c|}
\hline & Right eye & Left eye & Mouth & Face \\
\hline A & 0.004943 & -0.003944 & 0.001373 & -0.000195 \\
\hline A's picture & 0.007081 & 0.002083 & -0.000734 & 0.001185 \\
\hline
\end{tabular}

Table 12. Sum of the variance

\begin{tabular}{|c|c|c|c|c|}
\hline & Right eye & Left eye & Mouth & Face \\
\hline A & 0.192957 & 0.115114 & 0.013471 & 0.28218 \\
\hline A's picture & 0.234716 & 0.229761 & 0.024294 & 0.148688 \\
\hline
\end{tabular}

\section{Examination}

The variance was low in proposed method of the program better than previous research by these test results. Therefore, our method suppress variations in the result of the inter-frame difference, and the difference between current frame and before frame was small better than previous research. It is thought that our method suppress the facing direction in detection window by compensating the main spindle position of face. And we can see that the result of difference in entire face was improved better than other parts by packaging our method. It is thought that the entire face is more susceptible to the influence of face inclination than other parts. But the result of mean was almost no change between previous research and proposed method. It is thought that our method can't perfectly suppress the change of the main spindle position of face.

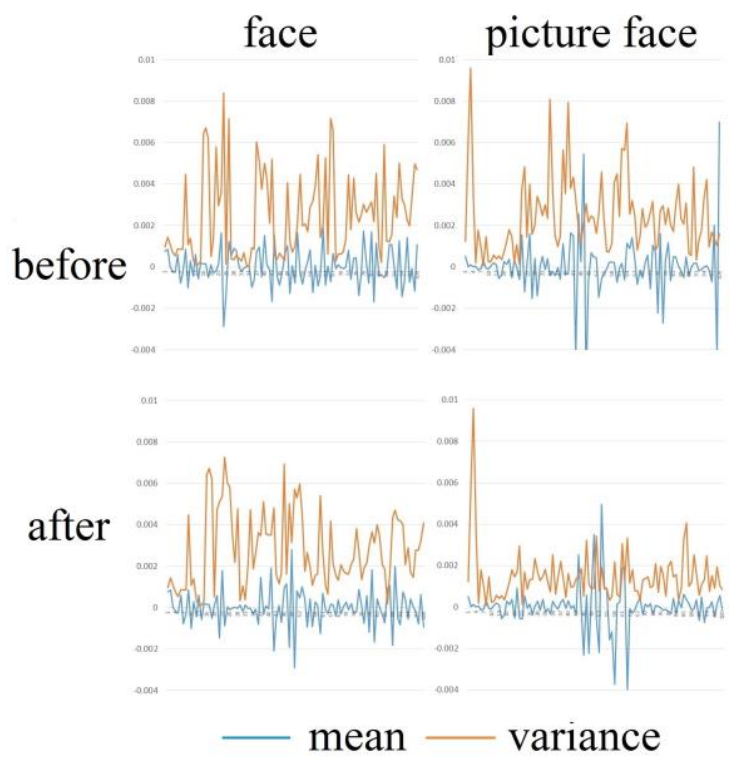

Fig.9 compare before and after 
The sum of variance of picture was almost same as the face's one in previous research, and it is difficult to distinguish the real face from the face picture. We show the comparison result in entire face between previous research and proposed method. From these findings, we can see that the sum of variance in entire picture face is lower than face's one. Therefore, we can discriminate between face picture and face, and the graph show that they have a difference which is capable discriminating picture face or face.

\section{Conclusion}

We proposed an approach of discrimination picture face and real face by compensating the main spindle position of face, discrete cosine transform and taking a difference between current frame and before frame. Previous research ${ }^{(4)}$ have difficulty in discriminating picture face and real face when the face picture is moving, but the proposed method modified the main axis of face which can discriminate the living real face and face picture. Fig. 9 shows that detecting fake face system is improved by our research.

The difference result of entire face gives the lowest results by using the proposed method which compensating the main spindle position of face when we move the picture. Therefore, we should consider that our method focus on an entire face. And we think that the discrimination with a variance is better than the mean value from those results.

In future, we are planning to implement the proposed discrimination system to the existence product to prevent the illegal authentication happening.

\section{References}

(1) HIROSHI KONDO, ZHIMEI YANG, TAKAHARU KODA and LIFENG ZHANG, "Sign only Synthesis" INFORMATION Volume 13, Number 3(B), pp.10271038,2010

(2) Lifeng Zhang and Hiroshi Kondo, "Innovating Sign Selection for Face Identication using DCT Sign" INFORMATION Volume 10, pp.101-110, 2007

(3) Paul Viola, Michael Jones, "Rapid object detection using a boosted cascade of simple features" ACCEPTED CONFERENCE ON COMPUTER VISION AND PATTERN RECOGNITION, 2001

(4) Syuuhei Kuroki, "Detecting fake face input for face authentication by DCT" Graduation thesis in Kyusyu Institute of Technology, 2014 